\title{
Inelastic transitions and counterflow tunneling in double-dot quantum ratchets
}

\author{
V. Moldoveanu ${ }^{1}$ and B. Tanatar ${ }^{2}$ \\ ${ }^{1}$ National Institute of Materials Physics, P.O. Box MG-7, Bucharest-Magurele, Romania \\ ${ }^{2}$ Department of Physics, Bilkent University, Bilkent, 06800 Ankara, Turkey
}

(Received 28 June 2010; revised manuscript received 10 September 2010; published 10 November 2010)

\begin{abstract}
The ratchet regime of unbiased double quantum dots driven out of equilibrium by an independently biased nearby detector has been theoretically studied using the nonequilibrium Keldysh formalism and the randomphase approximation for the Coulomb effects. When the detector is suitably biased the energy exchange between the two systems removes the Coulomb blockade on the double dot via inelastic interdot tunneling. The energy detuning determines whether the current flows in the same direction as the driving current (positive flow) or in the opposite direction (electronic counterflow). In both cases the intradot transitions lead to negative-differential conductance. Besides the ratchet contribution to the current we also single out a Coulomb drag component.
\end{abstract}

DOI: 10.1103/PhysRevB.82.205312

PACS number(s): 73.23.Hk, 85.35.Ds, 85.35.Be, 73.21.La

\section{INTRODUCTION}

Experimental schemes allowing the detection and counting of electrons tunneling through quantum dot systems have rapidly evolved once their importance to the solid-state spintronics was recognized. ${ }^{1}$ The inherent charge sensing effect $^{2,3}$ allows one to resolve tunneling processes through a quantum dot by monitoring the current characteristics of a nearby quantum point contact (QPC). Besides the "reading" operation, the Coulomb interaction between the detector and the measured quantum system mediates more subtle processes which together contribute to the so-called "detector backaction." For example, a large bias applied on the detector may induce transitions between the states of the measured system and alter therefore just the state one tries to "read."4

The direct (Heisenberg) backaction ${ }^{5}$ is due to the electronelectron scattering while the indirect backaction is attributed to the cross-talk of the two systems via their common bosonic environment. In two recent experiments Gustavsson et $a l .{ }^{6}$ and Gasser et al. ${ }^{7}$ investigated the electronic transitions induced in a double quantum dot by the absorption of photons or acoustic phonons from the environment whose properties are changed by currents passing through nearby QPCs. In a rather similar system Harbusch et al. ${ }^{8}$ also found a phonon-mediated QPC backaction. It was pointed out that the charge fluctuation in the detector are not entirely transmitted to the leads but also directly to the double-dot system. ${ }^{9}$

On the other hand the inelastic transitions induced by a biased detector remove the Coulomb blockade in the system to which it is coupled. In this case the detector acts rather as a current amplifier. Such an example is the double-dot quantum (DQD) ratchet considered in the experiment of Khrapai et al. ${ }^{10}$ The experiment revealed that a strongly biased QPC induces a current in the DQD having an internal asymmetry, even if the elastic tunneling is suppressed. The spatial asymmetry is due to the detuning of the two QDs which is controlled by gate potentials applied to each dot. It was argued that a finite current passes the double quantum dot when the energy absorbed from the detector matches the detuning energy. Another type of semiconductor ratchet was realized some time ago by Linke et al. ${ }^{11}$ (for a review on classical and quantum ratchets see Ref. 12).

The electronic transitions induced in quantum-dot systems by nearby biased detectors were previously studied within the master-equation approach and mostly in the context of continuous measurement of a closed qubit by a QPC..$^{13-15}$ Snyman and Nazarov ${ }^{16}$ used instead the extended Keldysh formalism and calculated the qubit transition rate. In a recent paper the master-equation approach was employed by Ouyang et al. ${ }^{17}$ in order to compute the detector backaction on open double quantum dots. The calculation presented in this work accounts for the interaction effects on interdot transition absorption and emission rates. However, in the implemented master equation the tunneling rates between the dots and the leads are noninteracting quantities. ${ }^{18}$ The model also assumes single-level quantum dots and therefore the intradot transitions or Coulomb repulsion are not included.

The aim of this paper is to provide a more involved theoretical description of Coulomb-mediated transport in a double-dot ratchet coupled to a charge detector. In our approach the various electron-electron interactions are treated on equal footing and therefore all the tunneling processes are affected by Coulomb interactions. Following the setup of Ref. 10 we calculate the current through the double dot for different detuning configurations over a large range for the bias applied to the detector.

Our method relies on the random-phase approximation (RPA) for the Coulomb interaction and on the nonequilibrium Keldysh formalism for interacting transport. It was established in a previous work ${ }^{19}$ and used in the study of the Coulomb drag effect in parallel quantum dots. ${ }^{20}$

The rest of this paper is organized as follows. We describe the model and give the relevant equations of transport theory in Sec. II. The numerical results are discussed in Sec. III which are followed by concluding remarks in Sec. IV.

\section{MODEL AND THEORY}

In the present work we use the partitioning approach to mesoscopic transport. ${ }^{21}$ The Hamiltonian splits in a part describing noninteracting and disconnected systems [i.e., DQD, 
detector (D), leads (L)] and a second term which includes the coupling to the leads and the Coulomb interaction,

$$
H(t)=H_{\mathrm{DQD}}+H_{\mathrm{D}}+H_{\mathrm{L}}+\chi(t)\left(H_{\mathrm{T}}^{\mathrm{DQD}}+H_{\mathrm{T}}^{\mathrm{D}}+H_{\mathrm{I}}\right) .
$$

The switching function $\chi(t)$ vanishes in the remote past and reaches a constant value in the long-time limit. The currents are calculated in the steady-state regime when all transients and initial correlations are safely neglected. In view of numerical calculations a lattice Hamiltonian will be used. We shall consider that each quantum dot contains up to two electrons. Although this is still a simple model it captures the effect of inelastic transitions between the two dots on the transport properties. The creation/annihilation operators on the site $n$ of the DQD are denoted by $c_{n}^{\dagger} / c_{n}$. By convention the sites $i=1,2$ belong to $\mathrm{QD}_{1}$ and $i=3,4$ to $\mathrm{QD}_{2}$. $H_{\mathrm{DQD}}$ then reads $(\langle$,$\rangle denotes nearest-neighbor summation),$

$$
H_{\mathrm{DQD}}=\sum_{i} \sum_{n \in \mathrm{QD}_{i}}\left(\varepsilon_{n}+V_{i}\right) c_{n}^{\dagger} c_{n}+\sum_{\langle m, n\rangle}\left(t_{m n} c_{m}^{\dagger} c_{n}+\text { H.c. }\right),
$$

where $V_{i}$ is a constant added to the onsite energies $\varepsilon_{n}$ simulating a gate potential applied on $\mathrm{QD}_{i}$ and $t_{m n}$ are hopping constants. The four semi-infinite one-dimensional leads are characterized by the same hopping constant $t_{L}$ (for simplicity the on-site energy of the leads, double dot, and detector are considered to be zero),

$$
H_{\mathrm{L}}=t_{L} \sum_{\alpha} \sum_{j=0}^{\infty}\left(d_{j_{\alpha}}^{\dagger} d_{j+1}+\text { H.c. }\right), \quad \alpha=L, R, l, r,
$$

where $d_{j_{\alpha}}^{\dagger}$ creates an electron on the $j$ th site of the lead $\alpha$. The double dot is connected to the leads $L, R$ (left, right) and the detector to $l, r$. Each lead is characterized by its chemical potential, the two biases applied on the DQD and detector being given by $V_{\mathrm{DQD}}=\mu_{L}-\mu_{R}$ and $V_{\mathrm{D}}=\mu_{l}-\mu_{r}$. The tunneling Hamiltonian between the leads and the double dot has the standard form,

$$
H_{\mathrm{T}}^{\mathrm{DQD}}=v_{L} c_{1}^{\dagger} d_{0_{L}}+v_{R} c_{4}^{\dagger} d_{0_{R}}+\text { H.c. }
$$

where $v_{L, R}$ are hopping constants between the dots and the nearest sites $0_{L}, 0_{R}$ of the leads.

The detector is also described as a four-site onedimensional chain, its creation and annihilation operators being denoted by $a_{k}^{\dagger}$ and $a_{k}$,

$$
H_{\mathrm{D}}=\sum_{\left\langle k, k^{\prime}\right\rangle}\left(t_{k k^{\prime}} a_{k}^{\dagger} a_{k^{\prime}}+\text { H.c. }\right) \text {. }
$$

The tunneling Hamiltonian $H_{\mathrm{T}}^{\mathrm{D}}$ is quite similar to $H_{\mathrm{T}}^{\mathrm{DQD}}$ given in Eq. (4) and we therefore omit to write it here. The last term $H_{\mathrm{I}}$ describes the various Coulomb interactions between electrons localized on different sites from the double dot and detector,

$$
\begin{aligned}
H_{\mathrm{I}}= & \frac{1}{2} \sum_{m, n \in \mathrm{DQD}} W_{0, n m} c_{n}^{\dagger} c_{n} c_{m}^{\dagger} c_{m}+\sum_{n, k=1}^{4} W_{0, n k} c_{n}^{\dagger} c_{n} a_{k}^{\dagger} a_{k} \\
& +\frac{1}{2} \sum_{k, k^{\prime} \in \mathrm{D}}^{4} W_{0, k k^{\prime}} a_{k}^{\dagger} a_{k} a_{k^{\prime}}^{\dagger} a_{k^{\prime}},
\end{aligned}
$$

where, for example, $W_{0, n m}=U /\left|r_{n}-r_{m}\right|$ is the bare interaction potential depending on the strength parameter $U$ and on the positions of the sites $n, m$ in the double dot.

The currents through the double dot and detector are calculated using contour-ordered Green's function. After standard manipulations the current entering the double dot from the left lead is given by a closed formula,

$$
\begin{aligned}
J_{\mathrm{DQD}}= & \frac{e}{h} \int_{-2 t_{L}}^{2 t_{L}} d E \operatorname{Tr}\left\{\Gamma_{L} G^{R} \Gamma_{R} G^{A}\left(f_{L}-f_{R}\right)\right. \\
& \left.-\Gamma_{L} G^{R} \operatorname{Im}\left(\Sigma_{I}^{<}+2 f_{L} \Sigma_{I}^{R}\right) G^{A}\right\},
\end{aligned}
$$

where the trace stands for the sum over the sites belonging to the double dot and $\Gamma^{L, R}$ are matrices whose single nonvanishing element is the one associated to the contact sites, i.e., $\quad \Gamma_{m n}^{L}=2 \pi v_{L}^{2} \rho(E) \delta_{m 1} \delta_{n 1}, \quad \Gamma_{m n}^{R}=2 \pi v_{R}^{2} \rho(E) \delta_{m 4} \delta_{n 4}$. Here $\rho(E)=\theta\left(|E|-2 t_{L}\right) \sqrt{4 t_{L}^{2}-E^{2}} / 2 t_{L}$ is the density of states at the contact site of the lead. The sign convention for the current is such that $J_{\mathrm{DQD}}$ is positive if electrons flow from the left lead toward the double dot. The retarded $\Sigma_{I}^{R}$ and lesser $\Sigma_{I}^{<}$selfenergies are calculated within a random-phase approximation scheme adapted for nonequilibrium Green's function (the details are given in our previous works, Refs. 19 and 20). It must be mentioned that the Green's function and interaction self-energies are finite rank matrices restricted to sites from the double dot and detector; they depend on both biases $V_{\mathrm{DQD}}$ and $V_{\mathrm{D}}$. In particular, the imaginary part of the interacting self-energy contains the inelastic Coulomb scattering processes. The retarded Green's function is obtained from the Dyson equation. The occupation numbers for each dot are derived from the corresponding density of states, the latter being in turn given by the imaginary part of the lesser Green's function,

$$
N_{i}=\frac{1}{2 \pi} \sum_{m \in \mathrm{QD}_{i}} \int_{-2 t_{L}}^{2 t_{L}} d E \operatorname{Im} G_{m m}^{<}=\int_{-2 t_{L}}^{2 t_{L}} d E \rho_{i}(E) .
$$

We implement numerically the RPA scheme and compute the interacting self-energies and the Green's function. These quantities are subsequently used in Eqs. (7) and (8).

\section{RESULTS}

In this section we present numerical simulations of the electronic transport in our double-dot system. The relevant parameters to be varied are the bias $V_{\mathrm{D}}$ on the detector, the interdot tunneling which we denote by $\tau$ (i.e., $t_{23}=t_{32}=\tau$ ), and the two gate potentials $V_{1,2}$ applied to each dot. These potentials are used to control the charge configuration in the double dot, that is the number of electrons in each dot. Also, by varying $V_{1,2}$ one also changes the energy detuning, defined as (see Ref. 10) $\Delta=E_{m+1, n}-E_{m, n+1}$, where $E_{m, n}$ is the 

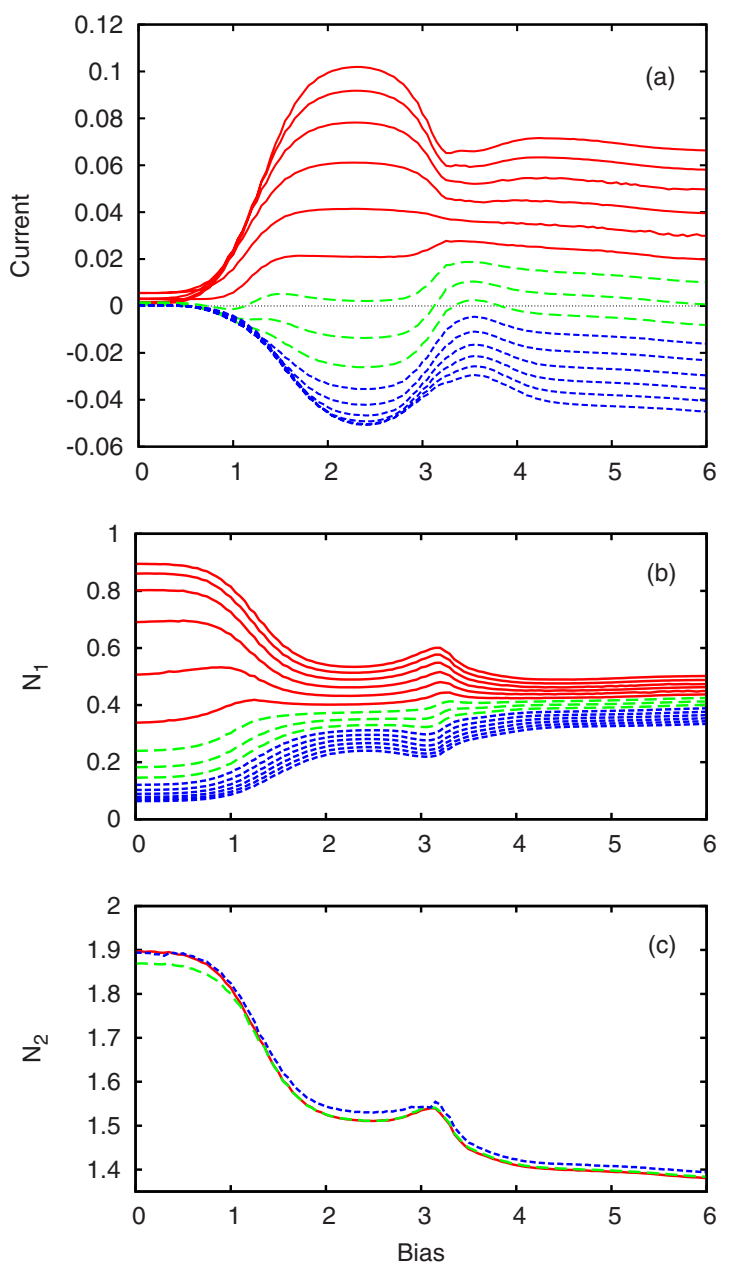

FIG. 1. (Color online) (a) The current through the double dot as a function of the bias applied on the detector for different values of $V_{1}$. There are three transport regimes: positive current-solid line, mixed-i.e., the sign of $J_{\mathrm{DQD}}$ changes long-dashed line, and negative current-dashed line. [(b) and (c)] The occupation numbers of the two dots for the same parameters as in (a). In Fig. 1(c) the values of $V_{1}$ are $0.4,0.6$, and 0.8 . Other parameters: $U=0.15, \tau$ $=0.1, v_{L}=v_{R}=0.35$, and $V_{2}=-1.55$.

ground-state configuration with $m$ electrons in $\mathrm{QD}_{1}$ and $n$ electrons in $\mathrm{QD}_{2}$. The intradot hopping parameter is denoted by $t_{D}$, and the current is given in units of $e t_{D} / \hbar$. The four intersite hopping parameters in the detector equal $t_{D}$ as well. The bias $V_{\mathrm{DQD}}$ applied on the DQD is very small and fixed, i.e., $\mu_{L}=0.01, \mu_{R}=-0.01$. The temperature $k T=0.001$. Also, for simplicity we consider identical quantum dots.

In Fig. 1(a) we show a set of curves for $J_{\mathrm{DQD}}$ simulating the outcome of a measurement which is easy to perform in common experimental setup. The gate potential $V_{2}$ is fixed such that as long as $V_{\mathrm{D}}=0 \mathrm{QD}_{2}$ contains almost two electrons (i.e., the two levels of $\mathrm{QD}_{2}$ are below the chemical potentials of the leads). Also, $\mathrm{QD}_{1}$ accommodates one electron.

The bias on the detector $V_{\mathrm{D}}$ is then varied over a large range for different values of the gate potential $V_{1}$ applied on $\mathrm{QD}_{1}\left(V_{1}\right.$ varies in the range $[0.4,0.8]$ with an increase step of $0.025)$. One can easily identify three transport regimes in Fig. 1(a). (i) In the first regime (see the solid line curves) the
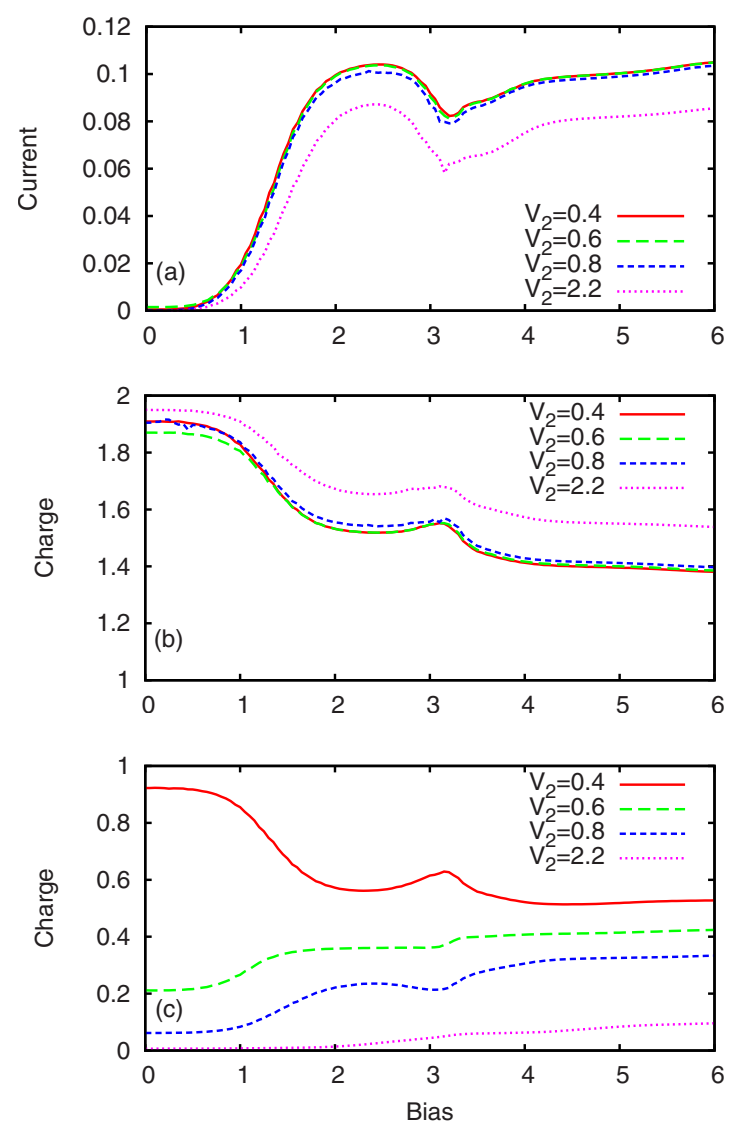

FIG. 2. (Color online) (a) The current through the double dot as a function of the bias applied on the detector for different values of $V_{2}$. [(b) and (c)] The occupation numbers of the two dots for the same parameters as in (a). Other parameters: $U=0.15, \tau=0.1, v_{L}$ $=v_{R}=0.35$, and $V_{1}=-1.55$.

bias $V_{\mathrm{D}}$ induces a positive current in the double dot. $J_{\mathrm{DQD}}$ first increases with $V_{\mathrm{D}}$ then decreases and saturates at larger values. One notices that a current appears in the DQD only around $V_{\mathrm{D}} \sim 0.65$. At this value the energy provided by the detector induces inelastic tunneling of electrons from both dots to the right lead. Indeed, both occupation numbers decrease in this regime [see Figs. 2(b) and 2(c)]. Notice that in the bias range [2.75:3.25], $J_{\mathrm{DQD}}$ decreases when the bias increases. This negative-differential conductance regime appears when the absorbed energy is spent on intradot transitions. If the energy absorbed from the detector matches the gaps between the levels of a quantum dot, electrons are excited on higher levels rather than to the leads or to the nearest dot. Indeed, one can easily convince himself that both occupation numbers increase in this case. As we have shown in our previous work on Coulomb drag, ${ }^{20}$ these intradot transitions compete with the interdot tunneling.

(ii) By further increasing $V_{1}$ (see the long-dashed curves) the current enhancement in the double dot reduces and the sign of $J_{\mathrm{DQD}}$ changes over the selected bias range. (iii) $\mathrm{Fi}$ nally, $J_{\mathrm{DQD}}$ takes only negative values (dashed curves), i.e., it flows against the driving current.

The mechanism behind each regime is revealed by the behavior of the occupation numbers $N_{1}, N_{2}$ as functions of $V_{1}$ and $V_{\mathrm{D}}$ shown in Figs. 1(b) and 1(c). Due to the weak inter- 
dot coupling the charge in the second dot is less sensitive to the variation in $V_{1}$ (one cannot actually discern the different transport regimes just by looking at $N_{2}$ ). It however drops from 1.9 to 1.38 over the bias range. It should be noted that $N_{1}$ has a similar behavior as long as it is larger than 0.5 [see the first four curves in Fig. 1(b) from top to bottom]. As the potential $V_{1}$ increases the occupation number of $\mathrm{QD}_{1}$ exhibits both qualitative and quantitative changes. While the levels of $\mathrm{QD}_{2}$ do not essentially depend on $V_{1}$ the levels of $\mathrm{QD}_{1}$ are pushed upwards, so the detuning between the dots increases. We see that in the third regime only a small amount of charge is localized on $\mathrm{QD}_{1}$ at $V_{\mathrm{D}}=0$, which means that its lowest level is now above the chemical potential of the leads. This also means that the detuning $E_{1,1}-E_{0,2}$ is positive and electrons inelastically tunnel from the highest level of $\mathrm{QD}_{2}$ to the lowest level of $\mathrm{QD}_{1}$. The scenario is confirmed by the numerical data, as $N_{1}$ increases while $N_{2}$ decreases.

We notice that a negative-differential conductance also exists in the ratchet regime. More precisely, electron occupying the lowest level of $\mathrm{QD}_{2}$ can gain enough energy to access the highest level of $\mathrm{QD}_{2}$ but this does not ensure that they would also tunnel to $\mathrm{QD}_{1}$ and to the left lead, as this process requires an energy amount already spent on the intradot transition. As a consequence the ratchet current decreases. By further increasing $V_{\mathrm{D}}$ (i.e., for $V_{\mathrm{D}}>3$, see Fig. 1) the processes leading to the ratchet current are again stimulated and the occupation number of $\mathrm{QD}_{2}$ decreases again. The ratchet current increases again and then saturates.

The third regime shown in Fig. 1(a) is therefore characterized by a negative ratchet current through the DQD which is entirely due to inelastic tunneling between the two dots. In this case electrons from $\mathrm{QD}_{2}$ can still tunnel from the highest level to the right lead but the level is more likely fed back from the same lead so a positive current is unlikely. In contrast, an electron that tunneled from $\mathrm{QD}_{2}$ to $\mathrm{QD}_{1}$ escapes more easily into the left lead because the weak interdot tunneling prevents relaxation in $\mathrm{QD}_{2}$.

In the intermediate regime the tunneling processes from $\mathrm{QD}_{2}$ to $\mathrm{QD}_{1}$ compete with the tunneling from the dots to the drain lead. When the two contributions (i.e., the positive and the counterflow currents) compensate each other the current through the DQD is very small, as can be seen in the first long-dashed curve in Fig. 1(a).

Another observation is that the negative ratchet current emerges at a larger (threshold) value of $V_{\mathrm{D}}$ than the positive current. This happens because in the latter case the minimal energy electrons need in order to escape into the drain lead is roughly given by the difference between their energy and the chemical potential of the drain even if at larger energies they could as well inelastically tunnel first from $\mathrm{QD}_{1}$ to $\mathrm{QD}_{2}$ and subsequently to the drain lead.

As the detuning increases the levels of $\mathrm{QD}_{1}$ are pushed above the bias window and the inelastic processes that lead to a finite current are the ones from $\mathrm{QD}_{2}$ to $\mathrm{QD}_{1}$. Obviously, the energy required for these processes increases with $V_{1}$.

Up to now we have seen that the inelastic interdot tunneling leads to a negative current through the DQD if the levels of the left dot are above the chemical potentials of the leads. In Ref. 10 this configuration corresponds to a positive detuning. What happens in the negative detuning case? We ana- lyzed this configuration as well by varying $V_{2}$ in the same range we previously varied $V_{1}$; the gate potential on $\mathrm{QD}_{1}$ is kept at $V_{1}=-1.55$. In this case $\mathrm{QD}_{1}$ contains almost two electrons when $V_{\mathrm{D}}=0$ while the lowest level of $\mathrm{QD}_{2}$ is below $\mu_{L, R}$ so $N_{1} \sim 1$. This time we find that $J_{\mathrm{DQD}}$ is positive over the entire range of $V_{2}$.

Figure 2(a) presents current curves for different values of $V_{2}$. Although they are quite similar, the occupation numbers $N_{1,2}$ depicted in Figs. 2(b) and 2(c) reveal again that there are different tunneling processes contributing to transport. For $V_{2}=0.4$ almost the same amount of charge is expelled from both dots in a similar way. The inelastic processes leading to the current are most likely the ones in which electrons tunnel from both dots to the leads. The setup changes at $V_{2}=0.6$ and $V_{2}=0.8$. As the lowest level of $\mathrm{QD}_{2}$ is shifted above the bias window (hence its occupation at $V_{\mathrm{D}}=0$ considerably decreases) the energy detuning becomes negative (i.e., $E_{2,0}$ $\left.-E_{1,1}<0\right)$ and the inelastic interdot tunneling from $\mathrm{QD}_{1}$ to $\mathrm{QD}_{2}$ activates at suitable values of $V_{\mathrm{D}}$. The comparison of the occupation numbers reveals some interesting features: (i) the excess charge of $\mathrm{QD}_{2}$ does not compensate the charge loss of $\mathrm{QD}_{1}$; (ii) the bias threshold value of $N_{2}$ increases with $V_{2}$, which means that larger energies are needed to populate the lowest level of $\mathrm{QD}_{2}$.

Actually at $V_{2}=2.2$ one observes that $\mathrm{QD}_{2}$ occupation is vanishingly small as long as the bias applied on the detector $V_{\mathrm{D}}<3$ and only slightly increases at larger values. This clearly means that the levels of $\mathrm{QD}_{2}$ do not participate in transport as they are pushed well above the chemical potentials of the leads by the gate potential and the energy absorbed from the detector is not enough to trigger significant inelastic tunneling from the lowest level of $\mathrm{QD}_{1}$ to the lowest level of $\mathrm{QD}_{2}$. Now, in spite of this fact the current through the DQD is not zero and the occupation number of $\mathrm{QD}_{1}$ decreases when increasing the driving bias. This means that electrons tunnel from the lowest level of $\mathrm{QD}_{1}$ to the right lead, without implying the levels of $\mathrm{QD}_{2}$.

These observations suggest that in the negative detuning case there are two contributions to the current, each one corresponding to different tunneling "paths" across the dot. On one hand electrons from $\mathrm{QD}_{1}$ can absorb energy and leave the double-dot system to the drain lead without populating first the lowest level of $\mathrm{QD}_{2}$ The current given by this " $\mathrm{di}$ rect" process is very similar to the Coulomb drag current in an unbiased single dot (see Ref. 20) and is not captured in previous approaches to the ratchet effect (Ref. 17). Indeed in the work of Ouyang et al. the Coulomb effects are only included in the interdot tunneling rates while the dotreservoir tunneling rates are noninteracting quantities-this means that an electron cannot tunnel from $\mathrm{QD}_{1}$ to the right lead unless it first inelastically tunnels to a higher level of $\mathrm{QD}_{2}$. As we have said, in our approach all the tunneling processes captured via the Green's-function formalism are affected by the interaction.

On the other hand, if the energy provided by the detector is large enough electron jump first to the higher level of $\mathrm{QD}_{2}$ and then relax to the right lead. This second contribution gives a ratchet-type current just as in the positive detuning case and becomes less important when $V_{2}$ increases. We find that $J_{\mathrm{DQD}}$ saturates for $V_{2}>2$. Note also that very little 

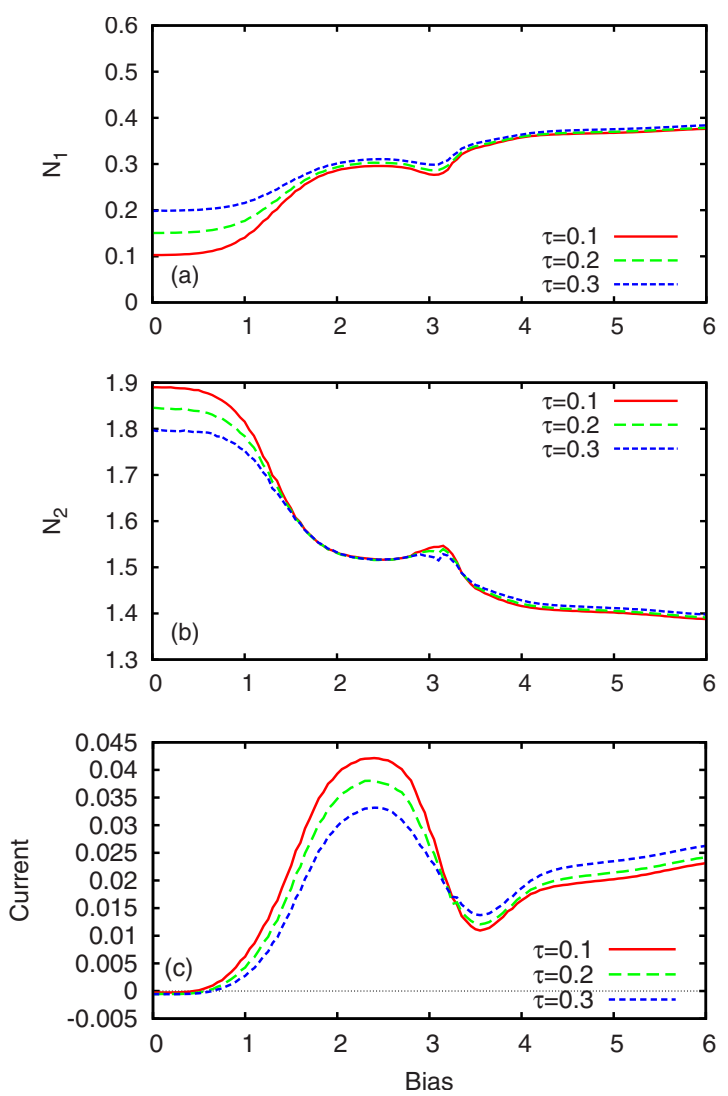

FIG. 3. (Color online) [(a) and (b)] The occupations of $\mathrm{QD}_{1}$ and $\mathrm{QD}_{2}$ as a function of the bias $V_{\mathrm{D}}$ at different values of the interdot coupling $\tau$. (b) The current $J_{\mathrm{DQD}}$ through the double dot. Other parameters: $U=0.15, V_{1}=0.7$, and $V_{2}=-1.55$.

charge adds to $\mathrm{QD}_{2}$ in this regime while $N_{1}$ still considerably decreases.

Let us point out that in our simulations the interdot coupling parameter $\tau$ is 3.5 times smaller than the coupling between the leads and the dots $v_{L}, v_{R}$. This does not guarantee that the electronic wave functions are strongly confined in one dot only. On the other hand in the experiments of Khrapai et al. the ratio between the interdot tunneling rate and lead-dot tunneling rate is $1 / 400$. In this regime the contribution coming from the direct tunneling could be probably neglected. However, we believe that at the theoretical level it is important to point out the possibility of this contribution.

In Figs. 3(a) and 3(b) we show the occupation numbers of $\mathrm{QD}_{1}$ and $\mathrm{QD}_{2}$ as a function of $V_{\mathrm{D}}$ for three values of the interdot coupling $\tau$. The gate potentials $V_{1,2}$ are chosen such that the ratchet carries a negative current. We observe that by increasing the interdot coupling more charge is localized on $\mathrm{QD}_{1}$ in the absence of the bias $V_{\mathrm{D}}$ but $\mathrm{QD}_{2}$ contains less charge. These changes are due to the shift of QD levels when $\tau$ varies. When $V_{\mathrm{D}}$ increases the effect of inelastic interdot transitions is still visible; $N_{1}$ increases while $N_{2}$ decreases but globally there is less charge added on $\mathrm{QD}_{1}$.

Then the ratchet current decreases at larger interdot couplings, as shown in Fig. 3(c) (note that we actually present $J_{\mathrm{DQD}}$ for clarity). A negative-differential conductance region is also present in this case; it appears when the energy absorbed from the driving system is rather spent on transitions
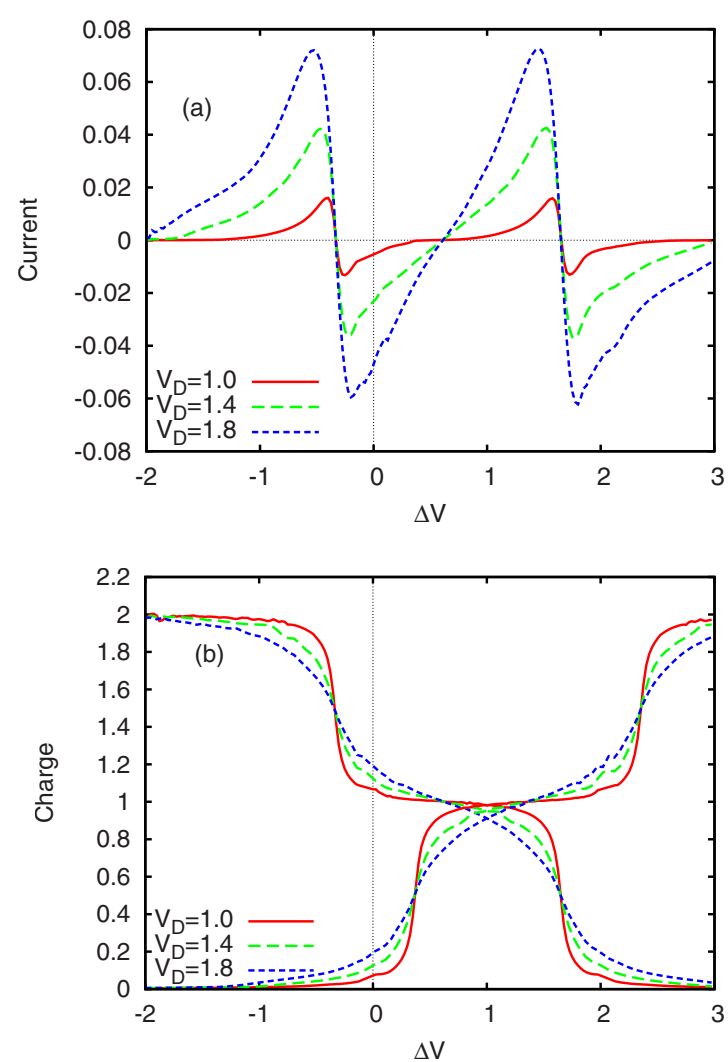

FIG. 4. (Color online) (a) The current $J_{\mathrm{DQD}}$ through the double dot as a function of the detuning potential $\Delta V$ for different values of $V_{\mathrm{D}}$. (b) The occupation numbers of the two dots. Other parameters: $U=0.15, \tau=0.1$, and $v_{L}=v_{R}=0.35$.

between levels in $\mathrm{QD}_{2}$. Indeed, around $V_{\mathrm{D}}=2.5$ the occupation number $N_{2}$ increases with the bias and then the charge transferred to $\mathrm{QD}_{1}$ decreases. The ratchet current is further enhanced when $V_{\mathrm{D}}>3.25$; the fact that $N_{2}$ decreases again suggests that inelastic tunneling from the lowest level of $\mathrm{QD}_{2}$ to the lowest level of $\mathrm{QD}_{1}$ becomes active.

Let us now investigate the role of inelastic interdot transitions to transport in a slightly different manner. Rather than keeping one of the gate potentials fixed we vary them simultaneously. More precisely, we redefine the gate potentials as: $V_{i}=V_{i}^{(0)} \pm \Delta V$, where $V_{i}^{(0)}$ are fixed such that at the lowest value of $\Delta V$ the charge configuration on the double dot is $\left(N_{1}=2, N_{2}=0\right)$.

By varying $\Delta V$ the levels of $\mathrm{QD}_{1}$ shift up while the ones of $\mathrm{QD}_{2}$ move down. The charge configuration evolves as follows: $\quad(2,0) \rightarrow(1,0) \rightarrow(1,1) \rightarrow(0,1) \rightarrow(0,2) \quad$ [see also Fig. 4(b)]. If the bias applied to the detector is small the two occupation numbers exhibit a steplike behavior as a function of $\Delta V$ (not shown). The transitions between steps correspond to the removal/addition of one electron from/to the corresponding dot. As expected, $J_{\mathrm{DQD}}$ is vanishingly small, the elastic tunneling being strongly suppressed because the QD levels are detuned and the interdot tunneling is small. The inelastic transitions within the DQD become active if a sufficiently large bias $\left(V_{\mathrm{D}} \sim 1\right)$ is applied to the detector. In Fig. 4(a) we present the current through the double-dot ratchet as a function of $\Delta$ for three values of $V_{\mathrm{D}}$. Clearly $J_{\mathrm{DQD}}$ has a sawtooth behavior and changes sign more than once. 
As $V_{\mathrm{D}}$ increases the steps of the occupation numbers soften, as a consequence of the inelastic transitions which now allow electrons to tunnel out to the leads. The steps are more affected at large values of $V_{\mathrm{D}}$ because the energy absorbed from the detector increases. One also observes that as $N_{1}$ passes through half-integer values $J_{\mathrm{DQD}}$ changes sign (from positive to negative).

The main point is that electrons from a level of $\mathrm{QD}_{1}$ which is below the chemical potential of the leads can tunnel into the drain lead without performing a transition to a higher level of the nearby dot (giving rise to a positive Coulomb drag current) or via an intermediate tunneling to a higher level of $\mathrm{QD}_{2}$ (a positive ratchet current). In this case the current in the double dot is positive, flowing in the same direction as the driving current. In contrast, when a level of $\mathrm{QD}_{1}$ passes above the bias window electrons can access this level from the lowest level of $\mathrm{QD}_{1}$ (performing an intradot transition) or from a lower level of $\mathrm{QD}_{2}$. As explained previously, the relaxation process from $\mathrm{QD}_{1}$ to the left lead is more favorable so the current is negative, i.e., it flows against the driving current. We remark that the amplitude of the negative current is always smaller than the one of the positive current.

In order to further substantiate the above discussion we present in Fig. 5 the double-dot density of states for two values of $V_{\mathrm{D}}$. The selected energy range illustrates the evolution of the double-dot states. The traces with positive (negative) slope correspond to $\mathrm{QD}_{1}\left(\mathrm{QD}_{2}\right)$. The bias applied on the detector leads to finite density of states above the chemical potentials of the leads attached to the DQD (we recall that $\mu_{L, R}= \pm 0.01$ ). This fact confirms the existence of inelastic tunneling processes induced by the driving bias $V_{\mathrm{D}}$. All the results presented here were consistently recovered for other values of the interaction strength.

\section{CONCLUSIONS}

We have studied the steady-state transport properties of a double quantum-dot system coupled capacitively to a charge detector. The Keldysh formalism and the random-phase approximation of the Coulomb effects provide a reliable description of the correlated transport. A current passing through the independently biased detector drives a current in the nearby double dot via energy/momentum exchange. We have calculated and discussed the inelastic current generated in the double dot as a function of the gate potentials applied on each dot. These potentials allow experimental control of the energy detuning. It turns out that the sign of this current depends on the energy detuning between the dots. We explain this fact by analyzing the various Coulomb-induced inelastic transitions within the double-dot system. We find (a)

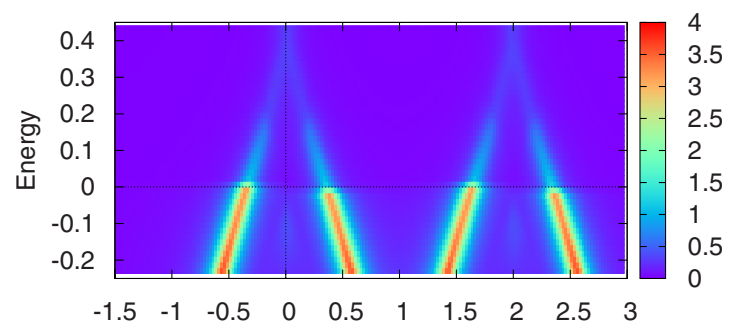

(b)

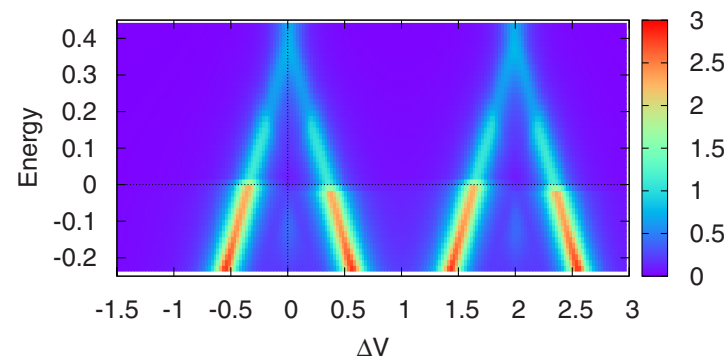

FIG. 5. (Color online) The density of states in the double dot as a function of the detuning parameter $\Delta V$ for two values of the bias applied to the detector (a) $V_{\mathrm{D}}=1.4$ and (b) $V_{\mathrm{D}}=1.8$. The traces with positive (negative) slope correspond to $\mathrm{QD}_{1}\left(\mathrm{QD}_{2}\right)$. Other parameters: $U=0.15, \tau=0.1$, and $v_{L}=v_{R}=0.35$.

that besides the inelastic interdot transitions leading to the ratchet current, electrons also inelastically tunnel from the dots to the leads without performing first a transition to a higher level of the nearby dot. This contribution to the total current could be important if the interdot coupling is not extremely small so that the wave functions are not highly localized on one dot only. We also report on the negativedifferential conductance regime associated with intradot transitions. These features can be experimentally confirmed.

The role of the interdot coupling is also discussed. The results obtained in this work are entirely due to the Coulomb interaction between the double dot and the detector and agree qualitatively with the experimental ones. ${ }^{10}$ More subtle effects due to the changes in the common bosonic environment not considered here would be of interest in future studies.

\section{ACKNOWLEDGMENTS}

B.T. acknowledges support from TUBITAK (Grant No. 108T743), TUBA, and EU-FP7 project UNAM-REGPOT (Grant No. 203953). V.M. acknowledges the financial support from PNCDI2 program 501 (Grant No. 515/2009) and Core Project (Grant No. 45N/2009). This work was also supported in part by TUBITAK-BIDEP. 
${ }^{1}$ S. Gustavsson, R. Leturcq, B. Simovic, R. Schleser, T. Ihn, P. Studerus, K. Ensslin, D. C. Driscoll, and A. C. Gossard, Phys. Rev. Lett. 96, 076605 (2006).

${ }^{2}$ M. Field, C. G. Smith, M. Pepper, D. A. Ritchie, J. E. F. Frost, G. A. C. Jones, and D. G. Hasko, Phys. Rev. Lett. 70, 1311 (1993).

${ }^{3}$ A. C. Johnson, C. M. Marcus, M. P. Hanson, and A. C. Gossard, Phys. Rev. Lett. 93, 106803 (2004).

${ }^{4}$ S. Ashhab, J. Q. You, and F. Nori, New J. Phys. 11, 083017 (2009).

${ }^{5}$ C. E. Young and A. A. Clerk, Phys. Rev. Lett. 104, 186803 (2010).

${ }^{6}$ S. Gustavsson, M. Studer, R. Leturcq, T. Ihn, K. Ensslin, D. C. Driscoll, and A. C. Gossard, Phys. Rev. Lett. 99, 206804 (2007).

${ }^{7}$ U. Gasser, S. Gustavsson, B. Küng, K. Ensslin, T. Ihn, D. C. Driscoll, and A. C. Gossard, Phys. Rev. B 79, 035303 (2009).

${ }^{8}$ D. Harbusch, D. Taubert, H. P. Tranitz, W. Wegscheider, and S. Ludwig, Phys. Rev. Lett. 104, 196801 (2010).

${ }^{9}$ It has to be mentioned that in order to discern phonon-mediated effects the double dot has to be asymmetrically coupled to the leads.

${ }^{10}$ V. S. Khrapai, S. Ludwig, J. P. Kotthaus, H. P. Tranitz, and W. Wegscheider, Phys. Rev. Lett. 97, 176803 (2006).
${ }^{11}$ H. Linke, T. E. Humphrey, A. Löfgren, A. O. Sushkov, R. Newbury, R. P. Taylor, and P. Omling, Science 286, 2314 (1999).

${ }^{12}$ P. Hänggi and F. Marchesoni, Rev. Mod. Phys. 81, 387 (2009).

${ }^{13}$ T. M. Stace and S. D. Barrett, Phys. Rev. Lett. 92, 136802 (2004).

${ }^{14}$ B. Dong, N. J. M. Horing, and X. L. Lei, Phys. Rev. B 74, 033303 (2006).

${ }^{15}$ X.-Q. Li, W.-K. Zhang, P. Cui, J. Shao, Z. Ma, and Y. J. Yan, Phys. Rev. B 69, 085315 (2004).

${ }^{16}$ I. Snyman and Y. V. Nazarov, Phys. Rev. Lett. 99, 096802 (2007).

${ }^{17}$ S.-H. Ouyang, C.-H. Lam, and J. Q. You, Phys. Rev. B 81, 075301 (2010).

${ }^{18}$ For the description of more subtle transport effects of Coulombcoupled mesoscopic conductors within the master-equation approach the tunneling rates of one system should depend on the occupation number of the nearby system, see, for example, R. Sánchez, R. López, D. Sánchez, and M. Büttiker, Phys. Rev. Lett. 104, 076801 (2010).

${ }^{19}$ V. Moldoveanu and B. Tanatar, Phys. Rev. B 77, 195302 (2008).

${ }^{20}$ V. Moldoveanu and B. Tanatar, EPL 86, 67004 (2009).

${ }^{21}$ C. Caroli, R. Combescot, P. Nozieres, and D. Saint-James, J. Phys. C 4, 916 (1971). 